\title{
LA FILOSOFÍA COMO AXIOLOGÍA
}

En los trabajos que he venido desarrollando como investigador de filosofía, tanto los que atañen a la temática inherente a la propia disciplina como los concernientes a la investigación sobre la investigación, manejo un concepto importante: la filosofía como axiologia. Ello implica no sólo el reconocimiento convencional de la filosofía como axiología, según el enunciado explícito del concepto, sino lo que pudiera ser también fundamental y controvertible, la acepción de la axiología en calidad de remate y culminación de la historia, con la pretendida tesis de que la axiologia es la única filosofia posible en esta época. El siguiente ensayo tiende a presentar las estimaciones más importantes en torno a este planteamiento que podría dar origen, obviamente, a un desarrollo mucho más amplio del que a continuación se le confiere.

Partiendo del problema que comporta el sentido de la filosofía como investigación cultural, obtenemos el criterio que resulta de observar dicho problema en la abigarrada pluralidad doctrinaria que contempla la historia, siguiendo el trayecto que significa la paulatina evolución del filosofar mediante el esfuerzo aporético de la cultura en su calidad de fundamento axiológico que desemboca en la crisis epistémica originada por la compatibilidad o incompatibilidad de sus doctrinas. Será necesario concretar el enfoque de la investigación sistemática señalando las grandes vertientes en que se desenvuelve la historia como expresión diacrónica del filosofar.

El problema que debe ser resuelto concierne primordialmente al concepto de filosofía, relacionado de manera estrecha con la investigación en virtud de la unidad que existe entre la postura sustentada y la tarea inquisitiva. Todas las definiciones de filosofía expresan en cada caso el concepto que se tiene de ella y a la vez corresponden al criterio sustentado por quien filosofa. Así, un partidario de la metafísica será en primer término devoto de la especulación; no reconocerá al filosofar como estudio de los hechos que se presentan en la experiencia ni como una metodología específica de las ciencias particulares, que son las dos grandes acepciones antagónicas a la metafísica. Recíprocamente, el empirista que se aferra a las manifestaciones positivas de la naturaleza no admitirá una filosofía caracterizada como "ciencia de las causas primeras y los fines últimos". Lógico es que el metafísico adopte la segunda definición y el empirista la primera, inherentes como son a sus respectivos sistemas. Análogamente, el axiólogo establece su postura en términos de valor, el existencialista acude a la experiencia vital, y así sucesivamente. De este modo se van definiendo en cada caso los respectivos campos de estudio. 
Vemos, pues, que el problema de la definición básica, o sea el establecimiento del axioma primario en cada doctrina, supone los requisitos que entran en juego para justificar la postura del investigador y los elementos constitutivos de la filosofía misma. Me propongo ahora inquirir si es posible superar la pluralidad doxográfica que, en cuanto insoluta divergencia de posturas, debe considerarse como un conjunto de opiniones subjetivas, o quizá como hipótesis de objetividad condicionada por las circunstancias heterónomas que influyen en el desarrollo del sistema, según los criterios de enfoque y las situaciones peculiares en que suele ubicarse una reflexión tan amplia y generalizante como la que nos ocupa.

\section{Búsqueda de universalidad}

Trascender la subjetividad tiene como base y finalidad el planteamiento de una exigencia fundamental de universalidad sistemática, o lo que equivale, de unidad totalizante. En tal virtud, la pluralidad doctrinaria se reduce a expresiones múltiples y contingentes de una secuencia desarrollada en forma inevitablemente particularista a través de las múltiples posturas que se manifiestan en la historia, aunque adquiere un sentido unívoco cuando se ubica en la trayectoria aporética mantenida como temática permanente a través de los sistemas y de las épocas.

El desenlace de este planteamiento radica en la hipótesis sincrética de que en el trasfondo de la evolución existe una sola filosofía, cuya dimensión diacrónica o histórica comprende las diversas modalidades que impone la cosmopsicovisión en cada etapa del devenir cultural, y en su dimensión sincrónica o sistemática se integra a la totalidad de la cultura como una de sus manifestaciones, y para decirlo mejor, la que viene a ser predominante al determinar el común denominador en el desarrollo de las polaridades axiológicas que se traducen en los bienes y formas de la cultura.

Por lo anterior, para acometer el tema expuesto, se procurará una definición que pueda interpretarse como mejor que las demás, fundando la idea de la filosofía, la cultura y la vida, en un concepto unitario y objetivo del valor, tendiente a realizar la cosmopsicovisión universal que constituye el desideratum del filosofar, aun con la relatividad que subsiste en el fondo de todo concepto, incluyendo la buscada universalidad del mundo y de la vida que a la postre viene a ser siempre contingente y relativa. De ningún modo podría ser definitiva la universalidad del valor, o en último término la considerariamos "definitiva" en el estado actual de las convicciones heurísticas, con una "definitividad" siempre hipotética y asintótica, nunca absoluta ni insuperable. En estas circunstancias, la perspectiva de asumir el concepto de universalidad como si fuera el único en la temática de cada época, permitirá presentarlo funcionalmente, según está dicho, como si fuera 
el único, tendiendo en ello a exponer la suficiente validez que permita atribuir a la filosofía el indispensable carácter de ciencia.

\section{Amplitud de la definición}

Este concepto es el que establezco o reconozco de la filosofía como axiologia, y conforme al mismo he conducido mis investigacionès bajo el supuesto de que, si no el único, es el mejor y más fecundo de todos los que se han formulado fundamentalmente en la época moderna. Ahora bien, para avanzar en el problema será necesario establecer una definición que corresponda al concepto expuesto en mis trabajos, cuya tesis he proyectado ahora en lo fundamental, para llevar adelante la investigación. Enunciaré brevemente esa tesis en una definición sumaria: filosofía es axiologia.

Como todas las definiciones, la anterior incluye el contenido sustancial del concepto definido y se traduce necesariamente en la postura que profeso; por consecuencia, señala también el camino que recorro en la materia. Pero la definición expuesta cuenta con una mínima extensión y puede llevarse a mayor amplitud para hacerla más abierta, de modo que comprenda explícitamente la connotación predicativa que, no obstante la brevedad con que se expone en este caso, incluye en todo su alcance el contenido de la definición propuesta. El concepto de filosofía se reduce inicialmente a una sola palabra: axiologia; lo que signifique la palabra misma requiere desde el primer intento una ampliación inmediata, aclarando que axiologia es la doctrina de los valores. A partir de este nivel predicativo puede llegarse a la disquisición más extensa que se quiera elaborar sobre los valores mismos, pudiendo desde luego ascender paulatinamente en nivel de generalidad. La definición anterior podría entonces ser sustituida por otra: la filosofía es la doctrina de los valores.

Como a su vez la filosofía no es la única disciplina que se ocupa de estudiar los valores, menester será precisar el punto de vista privativo que distingue al estudio filosófico del que acometen otras ciencias en relación con el tema; por ejemplo, la psicología, la sociologia, la historia, etc., que, ejerciendo los criterios correspondientes a sus implícitas perspectivas, pueden elaborar y de hecho elaboran extensas doctrinas particulares sobre el valor. ¿Cuál es, entonces, el punto de vista privativo de la filosoffa en relación a este problema? No es el de la psicología, que examina los valores en los parámetros subjetivos de la conducta; no el de la sociologia, que estudia los denominadores grupales en el desenvolvimiento colectivo; no el de la historia, que se interesa por precisar la génesis y evolución del valor en las diferentes etapas del devenir histórico.

El punto de vista privativo de la filosofía corresponde al criterio universal que plantea el problema de los valores en st mismos, definidos en 
tanto universales como criterios fundamentales de lo particular, los valores que valen con independencia de los individuos y los grupos que en ciertas condiciones específicas los acepten o rechacen; la filosofía pretende ubicarlos más allá de la evanescencia temporaria que suponen las épocas delimitadas en el devenir histórico. Estas cualidades del valor, que configuran el punto de vista filosófico, trascienden los hitos de la psicología, la sociología y la historia al sintetizarse en una categoría primordial que es la universalidad. Según veremos, ella constituye el punto de partida para comprender las cualidades que comportan sendas categorías fácticas del valor y configuran inmersas la categorética axiológica; nuestra definición quedaría entonces cifrada en los siguientes términos: la filosofia es la doctrina universal de los valores.

\section{La definición extensiva}

Por otra parte, la universalidad está directamente vinculada a la objetividad, en la medida que lo verdaderamente universal trasciende todo género de circunscripciones particulares y en primer término subjetivas, de donde la posibilidad de incorporar un nuevo elemento definitorio; se agrega la correspondiente nota fundamental a la definición anterior, evitando con ello cualquier confusión que pudiera interferir en los trabajos. La definición que ensayo ahora quedaría entonces como sigue: la filosofía es la doctrina universal y objetiva de los valores.

Como, por otra parte, los valores se realizan en la cultura, esta condición podría incorporarse al concepto inicialmente propuesto, en cuyo caso obtendríamos una nueva definición: la filosofía es la doctrina universal, objetiva y axiológica de la cultura; en ella, además de incluir el factum cultural que señala el requisito de realización en este campo, se remplaza al concepto teoria de los valores por su equivalente: doctrina axiológica.

Si además tenemos en cuenta la consideración expuesta sobre la exigencia de cientificidad, se justificará que incluyamos la connotación respectiva, ampliando un poco más la definición básica que entonces sería enunciada en los siguientes términos: la filosofia es la doctrina universal, objetiva, cientifica y axiológica de la cultura.

Ahora bien, sabemos que las teorías filosóficas se consagran en calidad de sistema, como una multiplicidad de conocimientos orgánicamente estructurados en torno a la unidad conceptual que determina su objeto, de suerte que un nuevo elemento ingresa a la definición: la filosofía es la doctrina universal, objetiva, cientifica, sistemática y axiológica de la cultura.

Podríamos continuar indefinidamente agregando notas predicativas a la definición, pues siempre es posible explicar un concepto mediante la incorporación de otros, y cada uno contribuirá a enriquecerla y hacer de ella una 
noción más explícita. Las notas predicativas representan conceptos susceptibles de elaboración ulterior y en conjunto se aplican a desentrañar su significado; cada enunciado puede, a su vez, incorporar otros más, configurando de esta suerte una extensa cadena predicativa que daría origen no sólo a una gran definición sino a extensos tratamientos que, desde diversos ángulos, serían mucho más prolijos y tenderían, sin embargo, a la misma finalidad definitoria, pues cualquier tratado sobre un cierto tema, por más extenso que se le suponga, no es otra cosa sino una amplísima definición del mismo, que acumula gran diversidad de conceptos y alimenta el enorme volumen de tratamientos que versan sobre una misma problemática, como en realidad sucede con la definición. Por ello, todo lo que se diga de la filosofía, todo lo que pueda agregarse a lo que ya se sabe e incluirse en la multitud de libros que se ocupan de ella, quedará comprendido en el inconmensurable alcance que tienen las tres palabras de la brevísima definición inicial: filosofía es axiología. Aceptando, claro está, que la filosofía se entienda realmente como axiología, como es mi caso.

La secuencia que acabo de exponer correlaciona las definiciones parciales, equivalentes por su significado aunque de distinto nivel en su extensión predicativa y pone de relieve la óptima posibilidad de ampliar el desarrollo de la investigación a partir de una célula tan pequeña como la primera definición propuesta: filosofía es axiologia; se trata de un juicio con mínima extensión $y$, sin embargo, entraña tal cantidad de posibilidades conceptuales que a partir de él puede y debe construirse un sistema de mayor alcance. Digamos que todo el sistema de la filosofía es la doctrina desenvuelta a partir de la orientación inicial que comporta el axioma primario de la postura correspondiente y cuya mecánica de extensión ha quedado expuesta.

\section{Omnipresencia del valor}

Debo ahora justificar por qué adopto el concepto del valor como norma fundamental en mis investigacaiones; a tal efecto partiré del principio regulativo que funciona en toda la historia $\mathrm{y}$ en todos los sistemas: el principio de universalidad, al cual me referi hace un momento. Ésta es la necesaria justificación para edificar cualquier sistema de conocimientos sobre el concepto más general que figura en dicho sistema. El principio en cuestión actúa en calidad de axioma, postulado o fundamento de toda una filosofía incorporada en el sistema de los valores, que es o debe ser hipotéticamente toda la filosofía, si llevamos el principio de universalidad epistémica a su última consecuencia, que consiste en subsumir toda su problemática bajo el alcance predicativo del axioma fundamental, según permita estimarlo la dirección aporética representada por dicho sistema.

Mencionaré algunos ejemplos de los más conocidos que verifican esta 
tesis, en la inteligencia de que la ejemplificación podría ampliarse eligiendo entre cualesquiera de los sistemas producidos; por ejemplo, la teoría de las ideas en la doctrina platónica, el concepto del ser abstracto en la aristotélica, la emanación hipostática del sistema plotiniano, la doctrina de Dios y de la Iglesia en el cristianismo, el entronque de la realidad en las ciencias que proclama el moderno empirismo, el cogito ergo sum y el método nihilógico en Descartes, la idea de mónada y fluxión cuantitativa en el atomismo leibniziano, la reflexión trascendental en Kant, y así sucesivamente. Cada uno de los sistemas que quieran mencionarse contiene a la base el principio más general de los que figuran en su recognición aporética, tendiendo en cada caso a convertirse en un principio universal.

De análoga manera, entiendo al valor como criterio representativo, no sólo de un punto de partida eficiente para desenvolver al sistema axiológico que, en cuanto tal, mantendrá básicamente el concepto del valor como entraña misma del sistema, sino también como la idea más amplia de cuantas hayan producido la filosofía y el pensamiento en general, pues a mi juicio esta idea es realmente universal y, a pesar de ello, no recae en las quimeras metafísicas, ilusiones subjetivas, particularismos o dogmatismos que son frecuentes en los intentos de cosmopsicovisión, los cuales deben darse por definitivamente superados $y$, en consecuencia, desterrados del panorama académico y científico del filosofar. Es, pues, el concepto del valor, el único que encuentro con la suficiente amplitud para considerarlo realmente universal y susceptible de fundar el más comprensivo sistema sincrético que englobe a todas las disciplinas y doctrinas filosóficas, con todas las ramas de la cultura cuyo conjunto integra el correlato funcional inherente a las determinaciones dialécticas de la axiologia; su dinámica supera los planteamientos anteriores del ser en cuanto los incluye a todos como modalidades axiofuncionales de la determinación ontológica.

Por ello creo que este mismo concepto es el único que asume la necesaria dimensión universal para incorporar en su seno las polaridades ontológicas, conceptuales e ideales que, en crecido número y variedad, constituyen la problemática del ser, del conocimiento, de la cultura, la vida y la filosofía misma, de suerte que, según el criterio esgrimido en la orientación que adopto para establecer la aporética metodológica, el concepto del valor es la base de una axiología universal y da origen al más fecundo sistema de cuantos se hayan construido, el que por modo eficacísimo garantiza la tarea tradidicionalmente propuesta a la reflexión: obtener un concepto del mundo y de la vida, la Welt-und-Lebensanschauung que yo designo con la palabra cosmopsicovisión. 


\section{Filogenia axiológica}

Para llegar a esta tesis he proseguido el que llamo desarrollo filogenético del pensamiento, o sea la evolución histórica examinada no únicamente en la secuencia temporal, como hace la historia propiamente dicha, sino mediante la integratividad doctrinaria que asume en cada una de sus grandes direcciones, según el orden de la extensión inherente a sus conceptos fundamentales, susceptibles de subsumirse a través de una relación múltiple e integrativa, comprensiva, sucesiva y unidireccional, de manera que el concepto menos extenso queda comprendido en los demás y el más extenso los comprende a todos. Prosiguiendo ese hito metodológico, he llegado a la conclusión de que el más amplio concepto que pueda obtenerse en la subsunción progresiva de los problemas filosóficos es el valor, por cuyo motivo establezco la genealogía histórica de las grandes direcciones que llevan a su determinación el nivel culminante de las doctrinas y los sistemas. La subsunción evolutiva reafirma de esta suerte al valor como etapa culminante en la filogenia axiológica y tiene el sentido capital que expondré simplificadamente a continuación.

Se trata de un criterio heurístico y completamente original que aplico desde mis primeros trabajos, en los cuales se contiene la programación filogenética de las dimensiones conceptuales que constituyen la base aporética y configuran la disposición flabeliforme de los temas respectivos. Esta disposición entraña la norma básica de la investigación progresiva y constituye el método más fecundo para desenvolver la pesquisa, al grado que permite no sólo excogitar la problemática tradicional de la filosofía, sino también la temática trascendental de la metafilosofía, o sea la hipotética disciplina que se ha llamado ocasionalmente filosofia de la filosofia y se desarrolla a partir de las pequeñas células empiriológicas y sus planteamientos iniciales, para llegar paulatinamente a los grandes desarrollos que proporcionan las mayores soluciones a los máximos problemas. El tema que ahora me ocupa corresponde a la idea direccional desenvuelta en el libro titulado Las grandes direcciones de la filosofia ${ }^{1}$ donde supongo y al mismo tiempo demuestro - punto de partida y de llegada- que la filosofía ensancha sus horizontes en cada etapa evolutiva con un nuevo planteamiento cíclico que engloba a los anteriores y los subsume en una perspectiva cada vez más amplia, hasta llegar al valor, que es para mí el más extenso de todos los planteamientos aporéticos; por consiguiente, deberá explicarlo y aplicarlo de la manera más comprensiva en cada una de sus etapas filogenéticas, o sea en cada una de sus implicaciones histórico-evolutivas.

Encuentro así que la primera fijación tética del pensamiento presocrático en el problema del ser, es determinada sobre la realidad inmediata con

1 Publicaciones de Dianoia, unam, Fondo de Cultura Económica, México, 1957. 
la inicial interpretación materialista-hilozolsta, pero se ve prontamente superada por la hipótesis del idealismo, toda vez que cualquier noción sobre la realidad es una idea, y recíprocamente, toda genuina idea parte de modalidades objetivas de la realidad; de ahí el consenso establecido que confiere mayor amplitud y profundidad a las doctrinas idealistas sobre las realistas. A su vez, la actividad ideatoria es una resultante del proceso cultural, producto y manifestación de la vida, se relaciona estrechamente con ella y depende de ella no sólo para la fijación de sus problemas, sino también al establecer los parámetros resolutivos que se reflejan en cada etapa filogenética de la historia ideal con las categorías constitutivas, precisamente, de la filosofia de la cultura; este nuevo enfoque filogenético incluye tanto al realismo como al idealismo, subsumiéndolos en cuanto etapas preparatorias y factoriales de la idea cultural, atendiendo al ritmo de una evolución doctrinaria que prosigue con la temática respectiva; llegamos a concluir que el anhelo clásico del filosofar, consistente en obtener un concepto del mundo y de la vida, resulta inaccesible si nos colocamos a espaldas de la actividad cultural. Por lo demás, esta conclusión es la misma que predomina desde el surgimiento de la filosofía moderna y se consagra en nuestro tiempo, mantenida por su propio peso en todos los denominadores fundamentales de la investigación contemporánea.

El valor, el ser y la vida

Considero que la filosofía de la cultura encuentra, a su vez, una nueva fase de subsunción filogenética en la filosofía de la vida, cuya denominación incluye tanto al vitalismo propiamente dicho como al existencialismo y demás modalidades del actualismo vital, que encuentran su apogeo en la época moderna con la autoconciencia crítica de la vida, pero cuyas doctrinas se originan sustancialmente desde la antigüedad. Supone esta corriente que, siendo el pensamiento y la cultura un producto de la vida, será en ella, en la vida, donde se localicen las esencias explicativas de la realidad; por efecto de esta tesis, las doctrinas vitalistas imperaron en época más o menos reciente como el último grito de la moda filosófica, aunque desembocan en el subjetivismo, principalmente en las doctrinas existenciales que registran la apoteosis de la subjetividad mediante la renuncia a toda clase de supuestos universales en aras de la moda momentánea y circunstancial que consiste, precisamente, en vivir el momento y la circunstancia.

Frente a esta multifacética posición vitalista, se constituye en el nivel teorético una etapa superior representada por los valores, en la medida que configuran ellos el denominador común de la cultura y la vida; para explicar esta superación es necesario reconocer que lo más importante de cultura y vida es precisamente el valor, puesto que vivimos una vida humana 
en la medida que la vida es cultura y se dirige en pos de los valores; o sea que lo medular en ambas, asi como en la filosofía que se ocupa de explicarlas, no radica en una mera descripción subjetiva de las vivencias temporarias, sino en la determinación objetiva y funcional de los valores que realizan, teniendo en sus más altos designios la consecución de universalidad que ha sido y sigue siendo la esencia peculiar de la filosofía.

Los valores son -válgase el pleonasmo- lo que más vale, lo único que realmente vale y lo único de lo cual vale la pena ocuparse para vivir y filosofar. La vida adquiere un sentido netamente humano en, por y con la realización de los valores, entendidos como funciones universales y expresiones particulares que el hombre promueve en su constante superación. Por ello, el concepto del valor es idóneo para conducir las investigaciones, no sólo en filosofía, sino en todo el campo cultural, y llega a las motivaciones ingénitas de la experiencia. Considero que el concepto del valor es el más amplio, extenso y fecundo de todos los conceptos, no sólo de la filosofía sino del conocimiento. Desempeña una función semejante a lo que fue en el prospecto metafísico la noción primaria del ser, o la no menos primaria del existir, y de hecho se coordina con ellas en la universalidad de la suprema extensión predicativa, porque todo lo que existe tiene sentido para nosotros en la medida que vale, de lo cual se infiere la identidad funcional y dinámica del ser y el valor: Todo lo que es, vale: todo lo que vale, es.

De ahí la insuperable amplitud que confiero al concepto material de la filosofía como axiologia, independiente de la función formal que asume en el mismo orden axiológico; considero en todo ello la tarea totalizante que desempeña la búsqueda de universalidad en la realización de sus denominadores particulares, proporcionando acceso a la cosmopsicovisión de todos los sistemas. La universalidad es, por antonomasia, universalidad del valor, y también son del valor sus manifestaciones particulares, por ello quiero destacar el sentido concluyente de esta meditación afirmando que: el ser es valor, la idea es valor, la cultura es valor, la vida es valor. Todo es valor: el valor es todo.

\section{Dialéctica del valor}

De las consideraciones anteriores se desprende que la noción objetiva y funcional del valor determina la postura que asumo en mis trabajos y el método que empleo para investigar. Reconozco en primer término a la objetividad del valor como base para desenvolver las reflexiones; la objetividad conduce a la universalidad, criterio por excelencia en que se funda tradicionalmente el empeño de la cosmopsicovisión, a la cual se llega en la conciencia crítica de las metátesis contemporánea que ha determinado el aspecto material del valor como la funcionalidad axiomática que se integra a la 
metodológica en el concepto formal del valor. Me parece que el concepto genérico del valor, entendido tanto en la acepción material como en la formal, es el más amplio y por consiguiente el más fecundo para justificar la temática concreta y simultáneamente el criterio metodológico de la investigación. Dicho concepto, y sólo él, ofrece la norma adecuada para desarrollar la tarea interpretativa de la cultura; a través de ella se aborda la cosmopsicovisión universal, después de haber asimilado sus funciones particulares al contenido empiriológico de las diferentes obras de la cultura y las disciplinas filosóficas fundamentales, como sucede con los planteamientos de la lógica, la ética y la estética que servirán dentro de un momento para ejemplificar el sentido dinámico de la axiología.

El primer nivel de observación indica que la creatividad del espíritu determina la inclusión del ser y el existir en la secuela omnicomprensiva del valor. En otras palabras, no sabemos lo que significan las cosas que son, ni los seres que existen, si los suponemos fuera del conocimiento, que a su vez entraña la consecución de la verdad como valor, entendida precisamente en el vínculo dialéctico del ser y el existir, como inclusión primaria de la vivencia en la esfera gnoseológica del concepto; esta dialéctica señala que el ser tiende al pensar y el pensar se justifica en el valor; lo que vale, vale por lo que es y lo que es, es por lo que vale. Lo que es y lo que vale se subsumen en el concepto de la verdad como valor.

Se trata fundamentalmente de un replanteamiento del viejo problema universal del ser en la correlación dialéctica del pensar, aunque ahora debe considerarse a través de la metátesis que provoca el surgimiento de la idea relativizada a la hegeliana manera en la dialéctica de la naturaleza y consagrada asimismo en la dimensión más amplia que registra actualmente el concepto universal, o sea la dialéctica del valor. De ahí se comprenderá también que la dialéctica axiológica es la más dinámica y comprensiva de todas las polaridades dialécticas, pues en ella se subsumen y explican lás modalidades del concepto en tanto funciones axiológicas.

A este problema se han ofrecido las más diversas soluciones, correspondiendo a la pluridimensionalidad operativa de las parcelas axiológico-aporéticas, o sean las modalidades primarias del valor que determinan al mismo tiempo las disciplinas creadoras de la cultura. En cada una de ellas se encuentra una justificación parcial del planteamiento axiológico y, ante esa multiplicidad axioconceptual, se impone una revisión casuística de cada disciplina para ascender a la normatividad crítica que fija la idea regulativa del valor, excogitada en un primer intento como denominador común de sus acepciones particulares, o sea como inducción axiológica que mantiene un fuerte resabio empirista en la medida que proporciona los materiales de la experiencia para obtener la buscada cosmopsicovisión universal. Sin embargo, más allá de esta asunción primaria se encuentra el fundamento teorético del 
denominador común que considero haber formulado correctamente en el concepto del valor como función, ${ }^{2}$ entendida la función en el sentido más amplio que significa relación o correlación, cuya fórmula primaria viene a quedar establecida por la ecuación general del conocimiento que expresa, de la manera más simple y al mismo tiempo más amplia, el planteamiento de la incógnita y la resolución del problema mediante una identidad axiomática de la forma $a=a$. Ésta es la tesis medular que sostengo en mis trabajos y señala el punto de convergencia en las directrices fundamentales de la filosofía crítica a partir de un apotegma radical que se podría expresar en los siguientes términos: todo ser es valor: toda filosofia es axiologia.

\section{El esquema axiocategorial}

La razón para considerar el ámbito universal del valor como base, esencial y objeto de la investigación, principalmente en el orden sistemático, estriba en el hecho de que al penetrar en los estratos axiológicos de las disciplinas culturales mediante la proyección que sobre ellas asume el método dialéctico, se alcanza la deseada universalidad de la cosmopsicovisión, en forma tal que puede verificarse dialécticamente a través del proceso inductivo-deductivo o a priori-a posteriori que denota el sentido dinámico de la metodología dialéctica en su aplicación deductiva, concreta y particular a la noción genérica del valor, obtenida a su vez mediante la asunción inductiva de las realizaciones específicas que adquiere en cada una de tales disciplinas.

Para desenvolver la tarea a partir de este principio, hay que tener en cuenta el esquema axiocategorial, o sea el esquema de las categorías axiológicas que se manifiestan en todos los valores y constituyen el denominador común fundamentante de la cultura, objetivada en la ingénita pluralidad de los facta específicos. Cuando hablo de un esquema axiocategorial quiero significar, de acuerdo al concepto clásico de esquema y de categoría, la organización sistemática de las propiedades inherentes a todo valor $\mathrm{y}$, por consiguiente, a todo ser, si genéricas se consideran, aunque derivan a formas particulares del valor y del ser, si de específicas se trata. En cuanto a que sea un esquema categorial el que representa dicha organización, la forma esquemático-sistemática se descubre y aplica en la tarea, derivada de la naturaleza misma de categoría, que es el esquema por excelencia, el concepto puro actuando como esquema simple del pensar; su validez podría parecer controvertible únicamente si desde su base rechazamos la conceptuación universal del valor, que equivaldría a rechazar el esquema general del conocimiento y el consiguiente esquematismo categorial significatorio del concepto; en última instancia, el esquema conceptual es método inherente a todo co-

2 Cf., mi trabajo La esencia del valor, en el $\mathrm{n}^{9} 6_{5}$ de la colección "Filosofía y Letras", UNAM, México, 1964 . 
nocimiento, y está necesariamente implícito en el proyecto que garantiza por su propia esencia el ejercicio de la autonomía epistémica en cualquier trabajo inquisitivo, por lo cual la fundamentación axiológica que estoy llevando a cabo en los niveles aporético, metodológico y sistemático, es también una fundamentación lógica y epistemológica.

Esta tesis me ha llevado a desarrollar la investigación conforme al instrumento criteriológico que considero de la mayor importancia; el concepto mismo de esquema formal-categorial y la función casuística que desempeña frente a la posición universal-genérica que ocupa la idea de una tolalidad material-funcional en la interpretación dialéctica de la polaridad conceptualaxiológica que corresponde en su correlación dinámica al inherente proceso creador y evolutivo de la cultura, lo cual equivale, en términos más simples, a sostener que todos los procesos culturales son creadores, dinámicos y evolutivos, y si no fueran procesos tampoco serían creadores, ni evolutivos, ni dinámicos.

En este punto llego a la parte medular del ensayo explicando en qué se funda el carácter sistemático de la axiología; el motivo que expondré ahora constituye la explicación genérica del método sistemático y resulta aplicable a cualquier caso de sistematicidad que, en cuanto tal, debe ser reductible a unidad; en este caso se trata de la unidad que determina el que designo como esquema categorial del valor, o sea el conjunto de categorias axiológicas que expresan los caracteres medulares de la creación cultural, las funciones que impulsan a la realización de los valores sobre las diversas direcciones estructurales en que se llevan a cabo.

El conjunto de dichas funciones constituye un sistema porque se agrupa alrededor de una propiedad fundamental y pretende agotar las posibilidades categoréticas del valor, o sea que las categorías expuestas deben ser sustancialmente exclusivas y todas las que pueden reconocerse en el problema comentado. Dicho sistema se encuentra infundido por el profundo sentido dialéctico que confiere una dinamicidad intrínseca al esquema categorial, y por consiguiente, a las realizaciones axiológicas de la cultura, que a su vez erigen el basamento aporético en la dialéctica de la investigación. Paso a exponer en qué consiste dicho esquema, dando por supuesto en todo su alcance el concepto de lo que significa en términos generales una categoría.

Me parece que la categorética axiológica es discernible mediante la concepción dialéctica de la tríada metodológica, aplicando las nociones generales que se encuentran en la teoría epistémica y constituyen por ello mismo una doctrina general del pensamiento y de toda forma creativa del espíritu. Dichas nociones generales nos indican que el conocimiento está determinado genéticamente por dos factores como son la realidad que presenta el objeto de conocimiento y la idealidad que encarna en el sujeto cognoscente; ambos factores se vinculan en la sintesis de la funcionalidad trascendental que tra- 
duce su correlación biunívoca. Creo haber encontrado además, que esta correlación se verifica en dos niveles o ciclos epistémicos, el que llamaré primario, donde figuran las tres categorías precitadas, realidad, idealidad y sintesis, y el segundo nivel que designo secundario, cuyas categorías son homólogas a las anteriores pero actúan en otra dimensión que permite reconocer su homología dialéctica; tal es el caso de las siguientes: concreción, polaridad y unidad que pasaré a explicar previa verificación del esquema respectivo.

\author{
ESQUEMA CATEGORIAL AXIOLÓGICO \\ Primer ciclo \\ realidad -idealidad \\ 'sintesis \\ Segundo ciclo \\ concreción -polaridad \\ unidad'
}

Significado categorial

Las dos primeras categorías del esquema - realidad e idealidad corresponden a los factores que se han reconocido tradicionalmente en el planteamiento epistémico, o sean la realidad del objeto conocido y la idealidad del sujeto cognoscente. La función que desempeñan estas categorías en la dinámica axiológica radica, en el primer caso, en recibir los determinantes del objeto, o sean las propiedades que lo hacen apetecible, en tanto que el sujeto proyecta las apetencias subjetivas en la idealidad, por cuya virtud se captan las propiedades objetivas en función de las valoraciones que postula el sujeto en su esquema vital.

Sabido es que ambos factores han ejercido gran influencia en la indoctrinación axiológica cuyo seccionamiento en objetivismo y subjetivismo corresponde a similar dicotomía que exhiben las doctrinas epistémicas. Pero así como en ellas existe una tercera categoría que es la síntesis dialéctica de las precedentes, ocupando por ello un plano superior, así también en materia axiológica existe una síntesis dialéctica trascendental en cuyo seno se establece la correlación de las funciones primarias que están connotadas en la objetividad y la subjetividad. De ahi que al incluir la categoría de síntesis en el esquema, no haga otra cosa sino reconocer el núcleo dinámíco de la titularidad axiológica, teniendo en cuenta que los valores no son aisladamente objetivos ni subjetivos sino que participan sintética y dialécticamente 
de ambas categorías primarias, incluyéndolas en los términos de la correlación biunívoca en la cual se localiza la función medular y dinámica del valor.

Obsérvese que en esta categorética axiológica se contempla la analogía estructural con respecto al esquema epistemológico, pues realismo e idealismo cognoscentes fueron trascendidos por el establecimiento de la dialéctica conceptual que los engloba y supera en aras de su biunívoca función vinculatoria. En la misma correlación se ubica y resuelve el problema tantas veces planteado del carácter teleológico, asintótico e infinito de los valores, que inequivocamente propugnan las posturas idealistas, frente al cual debaten las realistas la necesidad de consumarlos en la experiencia, atribuyéndoles el consiguiente carácter concretizante que en su extremo deriva al pragmatismo y al contingencialismo, oponiéndose, como es natural, a la normatividad teleológica que postulan los axiólogos idealistas; ambas funciones son comúnmente antagónicas, generando con ello el cúmulo de contradicciones que deterioran profundamente el contexto de la doctrina axiológica y su consiguiente definición como sistema científico. Ello será factible únicamente en el seno de la dialéctica trascendental que correlaciona funcionalmente las categorías precitadas y resuelve las unilateralidades en que suelen situarse las antagónicas doctrinas.

La polaridad es otra categoría del valor que tiene profunda repercusión en el tratadismo respectivo, toda vez que denota la existencia de valores $y$ contravalores, o sean las dos facetas contrapuestas de una misma entidad axiológica, como es el caso de las polaridades bueno-malo, verdadero-falso, bello-feo, etc. Obviamente, el sentido teleológico del valor exige la realización de su faceta positiva, pero la concreción señala inequívocamente la existencia de ambas modalidades.

Por ello, la concreción de los valores es la categoria que corresponde a la realidad en este segundo ciclo categorético-axiológico, pero se distingue de ella por la función especifica que asume como exigencia de realización, o sea que mientras la realidad se refiere a los valores dados en la realidad misma, concreción equivale a realización, al acto en el cual se generan las entidades axiológicas de acuerdo a las dimensiones casuísticas que envuelven a cada uno de los actos vitales. Esta categoría se puede expresar de la manera más amplia diciendo que el reconocimiento de un valor crea la obligación de realizarlo en la experiencia, aceptando que los valores no son entidades destinadas a una contemplación olímpica, sino a la realización concreta en la experiencia de acuerdo a los dictados que impone el sentido progresivo de la vida.

La categoría de unidad equivale a la unificación de los elementos formales que integran una estructura axiológica, lo cual comporta la organización sistemática del valor en las obras correspondientes. No podría escapar 
el valor a esta conspicua facultad, habida cuenta de que cualquier producto del espíritu contiene por su propia esencia la organización sistemática de los elementos generadores en cuya base radica invariablemente el principio de unidad. Por otra parte, no se trata sólo de la unidad intrínseca de cada modalidad axiológica, sino también de la más amplia unidad que agrupa a todos los valores, de suerte que esta categoría imbrica el postulado de la unidad integral del espíritu y la cultura, en cuyo seno debe realizarse el anhelo clásico de la cosmopsicovisión, el concepto universal del mundo y de la vida que es, ante todo, un concepto unitario y total.

De este modo puede observarse, una vez más, la dinámica de la filosofía que, considerada como axiología, depende fundamentalmente del paralelo asequible entre el problema planteado, el método que se aplique a resolverlo y el sistema que se construya con los aportes de su resolución. De no existir ese paralelo, el trabajo registrará las múltiples deficiencias, errores, contradicciones, heteronomías, unilateralidades, abstracciones, subjetivismos, etc., cuya menuda presencia demerita y contraviene el avance objetivo del pensamiento científico, de análoga manera a como sucede en todos los campos del saber.

\section{Problema, método y sistema}

Siguiendo el lineamiento general de la correlación dialéctica, la adopción del valor como punto de partida y criterio de prueba plantea por una parte el problema, y por la otra, proporciona el método y el criterio sistemático para su resolución. El enunciado de la postura axiológica es su justificación primaria y se manifiesta como sistema totalizante de máxima universalidad que engloba a las doctrinas históricamente dadas; el problema constituye también el principio del método para lleggar a la resolución sistemática, en cuya virtud se realiza en forma armónica y estructural, dependiendo a su vez de la posturã adoptada para resolverla, en directa vinculación al planteamiento del valor.

Nos situamos de este modo en el refrendo de la correlación problemamétodo-sistema que expuse anteriormente, al definir la condición básica que deberia satisfacer toda postura, independientemente de la circunscripción aporética que funciona como punto de partida y de llegada en el esquema categorial que corresponde a cada caso; dicho esquema constituye la condición universal de erección sistemática, en forma tal que el sistema se vierte a la integración del esquema formal correlativo, acogiendo la incorporación del problema que reconoce en cada caso como objeto por determinar.

Siguiendo el camino establecido en este principio, la base que reconozco para llevar a cabo la investigación sistemática, entendida como reflexión creadora, dependerá de la armonía y congruencia establecida en dicho esque- 
ma, aplicado integralmente tanto a la filosofía como a las ciencias de los valores; sabemos, por una parte, que la primera es un producto cultural, y por la otra, que la cultura misma es piedra de toque y criterio de verificación para el filosofar. Esta acepción y aceptación de la tarea reviste un sentido peculiar en la época actual, cuando se manifiesta una aguda crisis histórica en los valores universales y en las diferentes comunidades particulares, incluyendo a los individuos mismos. No se trata, pues, de una mera especulación sobre el sentido general del valor - lo cual sería bastante_, sino de la cuestión muy urgente que atañe a los valores específicos con carta ciudadana en las diferentes colectividades; pueden distinguirse en varias dimensiones, ya se trate de vastas regiones continentales, o de comunidades con una idio sincrasia homogénea, y en última instancia de toda la humanidad, que tiende asintóticamente a unificar sus valores genéricos en los términos de la asunción universal o cosmopsicovisión cuya pauta ha procurado obtener tradicionalmente la filosofía.

En estas condiciones, lo que signifique el valor constituye un tema que requiere con gran urgencia el acopio y la síntesis de todo género de tratamientos que se le han dado hasta ahora y los que se le puedan conferir en el futuro, satisfaciendo los requerimientos metodológicos que se presenten en un plano científico. En virtud de ello se rescata a la filosofía del viejo cargo que la considera como una reflexión abstracta, ya que lo concreto es la vida, y la dirección de la vida es el valor; nada más concreto puede haber en la vida que la concreción de la vida misma y lo más relevante de esta concreción es el valor. Tan efectiva como ella es la necesidad de orientación vital, para conferir a los valores un sentido de universalidad-concreta que apoye su relación permanente, progresiva, asintótica, dinámica y estructural. Filosofar equivale, en su acepción más concreta, a captar la vida en el momento en que la estamos viviendo; en su dimensión más amplia tiende a dirigirla hacia los grandes hitos de la evolución, que son también las grandes finalidades regulativas del desarrollo cultural en los bienes y obras que se manifiestan como normas de realización.

\section{El valor pragmático}

Los valores se comprenden generalmente como si fueran ideas prototípicas o normas ideales para la regulación de la vida. Esto es cierto; los valores, aun los tradicionales, no son simplemente las entidades puras y abstractas, inalcanzables y utópicas, que casi siempre se han entendido en algunas direcciones de la filosofía tradicional. Existen también las funciones concretas del valor y, como contrapolo de la tesis abstractiva, la doctrina pragmática ofrece una imagen que puede considerarse en cierto modo contraria, y en cierto otro, complementaria de la anterior. Para ella, los valores se encuentran en 
la realidad, son la entraña misma de los hechos que ocurren en la vida, de los mutables sucesos cotidianos que registran por partida múltiple sus variados matices y diversificadas polaridades.

Las divergencias doctrinarias que se han pronunciado en relación a los valores corresponden a una gran diversidad de matices proyectivos que se reflejan en la vida misma. Para los pragmatistas redicales se agota ella en la concreción individual de la cotidianeidad, de suerte que todos los actos adquieren un valor en sí mismos; en cambio, para los finalistas, que son mayoría, exhiben un sentido ulterior de raigambre universal y tienden a la realización de finalidades máximas que por naturaleza deben ser asintóticas. En el seno de la postura pragmática pueden llegar a identificarse como valores utilitarios, como por ejemplo, el bienestar o la felicidad, que se suponen realizados con plenitud en los actos consumatorios de cada especie. No figuran los valores pragmáticos a título de bienes infinitos o ideas regulativas, sino en cada caso se vierten a los hechos concretos e inmediatos que se agotan en la mutable existencia cotidiana. Por ejemplo, se habla de la felicidad ideal como paradigma hipostático que, a la platónica manera, es en el mundo prototípico un perfecto modelo normativo, aunque resulta verificable únicamente como una réplica de la idealidad en los limitados acontecimientos de la vida. Comprender el origen y sentido de esta pluralidad doctrinaria es necesario para integrar el sistema sincrético de los valores, sistema también que corresponde a la axiología considerada como filosofía.

La expresión más directa del pragmatismo axiológico se observa en las posturas así llamadas pragmáticas, que lo son en el doble sentido de postular valores finitos y reconocer su concreción en los actos que consumamos diariamente, confiriéndoles, en el caso extremo, una función utilitaria. Pero hay otras tesis que resultan de similar nivel aplicativo cuando afirman la consunción del ser y el existir en un instante de la vida. Ese instante puede ser cualquiera; no cualquier instante sino producirse en cualquier instante la apoteosis vital y el sentido de plenitud que acreditan el vitalismo y el existencialismo en todas sus épocas, con la peculiaridad inherente a cada expresión doctrinaria, coincidiendo en la misma convicción de viabilidad concreta y realidad prístina del valor consumado en el acto temporario de la existencia.

La importancia de las doctrinas concretizantes radica en que, en último término, lo concreto es inherente al valor así como a toda realidad y no se debe concebir ninguna teoria como si fuera contrapuesta a los valores concretos. Realidad y valor son términos coimplicantes, no se entiende uno sin el otro; por ello pierde sentido la abstracción cuando no tiene conciencia de sí y se manifiesta como una necesidad analítica, la cual debe ser reintegrada a la ulterior concreción sintética. En términos de concreción adquieren sentido las doctrinas que se afirman en cualquier modalidad de lo real, llámese acto, experiencia, vivencia, existencia, etc., denominaciones equiva- 
lentes en el seno de la realidad que, a fin de cuentas, es única y universal, mas no a la manera idealista sino en la paradójica función dialéctica que identifica la máxima universalidad con la máxima concreción: la plenitud suprema de la existencia se realiza y agota en la irreversible evanescencia de un instante preñado de lo absoluto. El valor pierde entonces la calidad de una idea infinita de regulación asintótica para ponerse al servicio de la plenitud vital, que en su hipóstasis mística es redención. El valor trasmuta la praxis en apocatástasis, el acto finito en acto absoluto; el deber ser se convierte íntegramente al ser.

\section{La axiología como ciencia}

En este vértice de la función axiológica en su dimensión pragmática ocurre la gran crisis del filosofar contemporáneo, cuyo nódulo radica en la posibilidad de establecer a la filosofía como ciencia, lo cual implica también la dimensión cientifica de la cultura. La función operativa y estructural del valor comporta la cientificidad en el deber de sujetarse a los datos de la experiencia y circunscribir a ella los términos de la reflexión, develando a la verdad como valor en el seno de la función trascendental que impone cualquier teoría moderna, siempre y cuando se reconozca su calidad científica como disciplina intelectiva.

Sabemos que la característica metodológica fundamental en la ciencia es su bipolaridad dialéctica: idealidad y realidad, abstracción y concreción, unidad y pluralidad; por ello reconocemos que la verdad no debe permanecer en el ámbito de la experiencia fragmentaria, como tampoco puede subsistir en el plano de la abstracción pura. El señalamiento de ambos requisitos es indispensable para comprender por qué la metodología dialéctica, bipolar y axiológica, oscila entre lo ideal y lo real, pero también se verifica en el tránsito de lo abstracto a lo concreto, y viceversa. Esa misma dialéctica reafirma la tesis que he adoptado de la tradición criticista, al sostener que la filosofía, en tanto axiología, es una filosofía de la cultura, la única filosofía posible, entendiendo por tal la que debe sustentarse en una base realista para evolucionar sincréticamente en paralelo al decurso histórico del desarrollo cultural.

Pero la misma concreción axiológica pone en crisis la estabilidad y autonomía de nuestra disciplina; teniendo en cuenta que la tendencia tradicional impele a la consideración abstracta del valor, la crisis se genera cuando nos proponemos verificar su concreción, porque entonces intervienen las disciplinas de la cultura como ciencias determinantes del valor; la axiologia corre el riesgo de convertirse integramente a las diversas ramas culturales y agotarse en la tarea que llevan a cabo sus disciplinas especificas, las cuales actúan también como ciencias auxiliares de la axiología filosófica y, en un momento 
dado, pueden interpretarse como ciencias axiológicas en sí mismas, incorporando los desarrollos que aún mantiene con cierta independencia la critificada axiología material, cuyo carácter filosófico parece estar cada vez más en entredicho; a medida que se incrementa la demanda de cientificidad, no sólo genéricamente para el filosofar sino específicamente para el valorar, se pone en tela de juicio que la tarea pueda ser acotada en los linderos filosóficos. De ahí la paulatina conversión que estamos contemplando de la filosofía material axiológica en ciencia material axiológica, ya que esta última desahoga con eficacia la tarea originalmente propuesta para la axiologia filosófica.

El resultado que deriva de reconocer en forma sustancial la concreción del valor, consiste en admitir la homóloga concreción que, por idénticas razones, concierne a la axiología y, si la entendemos biunívocamente como la filosofía "en sí misma", la única filosofía posible, podría quedar subsumida bajo la metátesis contemporánea que la erige en calidad de disciplina axiológica; en tales condiciones, la axiología material o científica, configurada en su más amplia acepción por todas las ciencias y ramas de la cultura, reclamará los fueros que orillarían a considerarla stricto sensu como una cieñicia independiente y particular, de análoga manera a como ha sucedido antes con las matemáticas, la física, la biología, la sociología, la psicología, la antropologia y demás especies dianoéticas que en una época fueron parte de la filosofía o se confundieron con ella, pero cuya recíproca evolución las apartó inicialmente al escindir sus respectivos problemas y las reunió más tarde en la función metodológica, siguiendo el proceso que he descrito como un esquema fusiforme, señalando como diorama del desarrollo alternante la temática científico-filosófica desenvuelta en una especie de movimiento pendular en correlación a las polaridades axiológicas de la ciencia y la cultura.

Me pregunto si en tal virtud y a pesar de ella, o precisamente por ella, la filosofía podrá seguir elaborando una "doctrina de los valores", o si la dilatada era en cuyo prolongado transcurso estuvo de moda concebir al valor en forma teleológica, pura o teorética, ya terminó, o si cualquier doctrina axiológica se reduce al conocimiento del valor tal como se genera en el perimetro de la concreción cultural, en cuyo caso estará sujeto a las demarcaciones de realización bajo el condicionamiento que suponen irrevocablemente las ciencias particulares.

La respuesta pudiera ser negativa, a pesar de que la axiología sigue constituyendo, a mi criterio, la única opción posible para conservar un atributo material a la filosofía, es decir, un objeto concreto del cual se ocupe en forma tan constructiva que si declaramos inadecuada esta opción habrá que suponerla desierta o buscar para ella otra encomienda de tipo formal, como efectivamente se está intentando en esta época. Para demostrar el sentido de la metátesis voy a centrar la atención incidentalmente en lo que sucede con 
los valores prototípicos, que han sido clásicos en la tradición y generan la temática de las disciplinas fundamentales: verdad, bondad y belleza.

\section{La verdad y la ciencia}

El valor verdad, considerado como contenido del conocimiento, constituye la forma axiológica del saber y el sustrato de la investigación científica; por ello se ha dejado al arbitrio de las ciencias particulares definir en qué consiste la verdad, desde que se descubrió su atribución exclusiva para edificar el conocimiento como un saber objetivamente demostrado $y$, por consiguiente, para formular la única verdad posible sobre los problemas que ellas manejan. Es un hecho que desde la entronización filosófica como autoconciencia crítica del saber, la antigua lucubración metafísica ha quedado definitivamente superada. ¿Tendría hoy algún sentido que viniesen los filósofos a predicar lo que es o deben ser la verdad y la ciencia, más allá del dictamen que a tal efecto emiten los investigadores amparados en la metodología demostrativa de las ciencias particulares?

Por ello tenemos que, ahora, quienes forjan la teoría de la verdad se atienen estrictamente a lo que demuestran las ciencias; los teóricos del conocimiento deben ser sus propios cultivadores: los hombres de ciencia. El concepto de la verdad se constituye irrevocablemente sobre la verdad misma, producida por las ciencias y teniendo a la vista sus ingénitas limitaciones; según la lógica moderna, los "filósofos de la ciencia", entendidos a la antigua manera, nada tienen qué hacer en el trabajo desenvuelto bajo los auspicios de la lógica cientifica, que es también y por consiguiente, la lógica de las ciencias, sobre todo cuando las matemáticas, que constituyen la ciencia por antonomasia, edifican racionalmente desde su primera base la estructura formal del saber llevando a consecuente desenlace la metodología rigurosa que prosigue los cánones estrictos de la predicación apodíctica fundada en la escuela metodológica de la formalización axiomática y consagrada en las posturas de raigambre matematicista que entronizaron a partir del racionalismo barroco del siglo xviı, cuya formulación más acabada es el actual neopositivismo lógico que atiende al estudio del conocimiento científico y construye la verdad, o el concepto de la verdad, según las significaciones materiales y semánticas de la judicación, traducidas metodologicamente a los enlaces formales de la conceptuación predicativa.

\section{La bondad y la conducta}

En el campo de la moralidad sucede otro tanto; la atribución filosófica original, consistente en postular de manera especulativa lo que debe ser la conducta, está convirtiéndose cada vez más en científica, por cuya sola 
virtud es posible explicar y fundar objetivamente el valor bondad aunque su rescate como contenido esencial de la conducta ha demorado más que en el caso anterior, debido al viejo distingo entre lo bueno y lo justo, entre la conducta moral y la conducta legal, quedando casi siempre la primera a cargo de la especulación filosófica, mientras la segunda se adjudica a la ciencia juridica, asistida por el compacto sistema de disciplinas sociales que la auxilian en todos sus aspectos para hacer de ella, precisamente, una ciencia $o$, más aún, todo un complejo de ciencias.

En medio de esta pronunciada dualidad de posiciones normativas frente al problema de la conducta, se mantiene la tendencia a asimilar lo bueno a lo justo, y viceversa, de modo que el imperativo categórico del deber ser impulsa no sólo a convertir la convicción personal en norma de universal observancia, sino en todo un sistema de acción jurídica que haga exigible por medio de la ley lo que señala como bueno la conciencia del individuo, tendiendo a erigir lo justo en valor material y formal de la conducta. Es necesario reconocer que el concepto de bondad ha evolucionado lo suficiente para superar a la escueta creencia subjetiva de lo que entiende cada quien como bueno, según antes se comprendía, a espaldas de lo fundado objetivamente como justo.

La dialéctica de la conducta y su correspondiente valor se establecen en la interinfluencia mutua de ética y derecho, ley individual y ley social, principio subjetivo y principio objetivo, imperativo hipotético e imperativo categórico; entre las dos posiciones que podríamos considerar radicales, o sea el extremo rigor de la jurisprudencia formal y la extrema liberalidad de la moral subjetiva, se construye el justo medio de la ética cientifica que, de la manera más amplia, comporta la normación concreta de la conducta individual y colectiva. La razón de tal distingo se encuentra llanamente en las limitaciones del derecho frente a la moral, o sea la imposibilidad de convertir integramente en ley todo lo que la conciencia moral señala como bueno.

Para superar el subjetivismo, y en última instancia el abstractismo que comporta el mantenimiento de una moral basada en la aceptación de lo "bueno", como seria la moral de las buenas intenciones y los buenos sentimientos, hay que reconocer la ineludible conformación educativa que imprimen a los individuos y los pueblos las circunstancias que se manifiestan a través de la educación, como son las influencias múltiples del medio ambiente, las tradiciones, la familia, las leyes, los ejemplos personales, etc., todo lo cual pudiera no coincidir en principio con la buena moral de las intenciones y los sentimientos puros. Existe a tal respecto el pluralismo ético, cuya dimensión histórica y sociológica nos enseña que lo bueno para unas sociedades o individuos pudiera no serlo para otros, pues el valor de la conducta, así en aquéllas como en éstos, depende de importantes factores sociológicos, biológicos, pedagógicos, históricos, jurídicos, psicológicos y antropológicos, 
que influyen en las convicciones personales o grupales y, en mayor o menor grado, las determinan, condicionando los sentimientos y las intenciones que en el consenso doméstico y en algunos pensamientos idealistas pretenden agotar las posibilidades de la ética en una moral buena, hermosa, impoluta y subjetiva.

Contrariamente a esta posición, que debe considerarse superada aunque todavia operante en el marco de las costumbres y convencionales sociales, Ia moderna ciencia de la conducta está remplazando cada vez más a la antigua moral subjetiva y dogmática, no sólo en el planteamiento de los principios que deben regir al comportamiento, en cuyo ámbito posee una definitiva ventaja sobre la ética tradicional, sino inclusive para procurar una favorable modificación tipológica de los individuos y los conglomerados, con objeto de que adapten su conducta a lo que señalan objetivamente las normas científicas, y lo hagan no sólo por deber, sino también por convicción, de suerte que su cumplimiento proporcione las íntimas satisfacciones que la ética clásica supone como inherentes al valor bondad, y cumpla asimismo las estipulaciones formales de las ciencias normativas en el variado orden jurídico, sociológico, histórico y antropológico que les concierne. Este es otro caso muy conspicuo donde la vieja filosofía especulativa pierde terreno frente a la ciencia del valor; el problema traslada su centro de gravedad a la demarcación concreta de los parámetros operativos que rigen en la conducta con el consiguiente menoscabo de la cada vez más trascendida ideación abstracta.

\section{La belleza y el arte}

El tercero de los valores clásicos que examinaré es el de la belleza; depara una perspectiva similar a la que se revela en los casos anteriores, o sea una creciente determinabilidad científica frente a la proporcional disminución especulativa, y por consiguiente, la depreciación de la filosofia del arte frente al incremento de la ciencia del arte. Es necesario precisar, ante todo, que hablar de belleza - para los efectos de este ensayo- es tanto como referirse específicamente a la belleza del arte $y$, aunque el dominio artístico se resiste mayormente a recaer bajo la conceptuación científica, lo cierto es que la belleza realizada en las obras de arte resulta asequible a través del análisis técnico y formal de las obras mismas, quedando al margen las especulaciones subjetivas que durante tanto tiempo alimentaron a la motivación estética y siguen imperando todavía en la llamada "estética filosófica". Allí se recogen como insostenibles abstracciones los temas que no encajan en la estética científica, sino que deben ser motivaciones personales por su propia índole subjetiva. Así, un literato o poeta estará abocado a hablar en primera persona de la literatura o la poesía, más que un filósofo propiamente dicho, quedan- 
do a cargo del crítico o el historiador del arte la tarea de llevar a cabo la excogitación científica de lo que significa su auténtico valor.

El filósofo del arte se encuentra sujeto al ineludible requerimiento de contemplar con mirada crítica, objetiva e imparcial, el verdadero sentido de la creación artística, restringiendo sus emociones a cambio de descifrar correctamente las ajenas. Y cuando digo filósofo del arte es para designar no solamente a quienes de manera profesional efectúan la meditación filosófica propiamente dicha, sino también al crítico y al historiador que, desde sus respectivas posiciones, emiten un juicio valorativo de las obras, en lo cual consiste la asunción axiológica que interesa destacar en este caso.

Para consumar la correspondiente tarea judicativa y valorativa se ponen en juego las categorías técnico-estéticas que privan en cada una de las artes y las obras. La belleza de un cuadro, por ejemplo, es discernible mediante el examen del tema que expresa, de las formas, la composición, el colorido, el dibujo, las proporciones, etc. Una obra literaria resulta asequible en términos de prosodia, sintaxis, métrica retórica y lo que es característico de todas las artes: temática y composición. Por su parte, la música tiene sus categorías específicas, como son la armonía, la melodía, la polifonía, la instrumentación, la orquestación, etc., incluyendo los citados coeficientes genéricos de temática y composición que resultan primordiales en todas las obras para realizar significativamente el valor de la belleza artística.

Dicho valor fue objeto, durante mucho tiempo, de las más diversas especulaciones en el campo de la filosofía tradicional; cada quien imponía un criterio sobre lo que a su juicio significaba la belleza, tanto en el arte como fuera de él, y primordialmente se resaltaba lo bello de la naturaleza y de los sentimientos. Ahora sucede lo contrario; el verdadero filósofo-estético es ante todo el crítico de arte que se sitúa frente a una obra para juzgarla y analizarla, poniendo en juego criterios cientificos y omitiendo en todo lo posible recaer en cualesquiera lucubraciones que puedan obstruir la severidad inherente al juicio estético, que es el juicio del valor en el arte. Sobre este camino se ubica la estética contemporánea, entendida como teoría de la belleza artística y, en tal sentido, apenas se distingue de la crítica de arte, o de la estética científica por el enfoque local y temporal de la segunda, a cambio del "concepto universal de la belleza" cuya obtención sigue siendo el propósito indeclinable de la primera. Pero este propósito ya no es asequible por medio de la especulación, sino mediante el sólido apoyo de la historia y la crítica de arte que, a su vez, encuentran una justificación plural en las aportaciones que a su respectivo campo brindan las ciencias del arte. Este es el material para la edificación de la estética, o sea para la comprensión del valor como belleza y de la belleza como valor; simultáneamente, del arte como fuente de valor y belleza. 


\section{Formalidad del valor}

De todo ello se desprende la intervención cada vez más amplia que tienen las ciencias particulares en la determinación de los valores y la paulatina exclusión que han estado llevando a cabo de la filosofía en cuanto doctrina "material" que tiende a establecer en forma concreta cómo debe ser su realización, asunto éste que compete directamente a los creadores de la cultura. Siguiendo el esquema matemático que se ha producido en todas las etapas significativas de la historia, observamos que la filosofía presenta una coyuntura análoga a los casos anteriores, y asume una vez más la tarea formal que le está reservada en cuya sola virtud se distingue de las ciencias particulares y puede coexistir sin contradecirse con ellas. Para comprobar esta tesis bastará con señalar las atribuciones reservadas a la filosofía dentro del panorama general que presenta la problemática del valor, y simultáneamente del ser, de la idea y la vida, en los términos de subsunción aporética que he señalado.

¿Cuáles son, pues, los problemas inherentes a la axiología formal-trascendental? En primer término, sale al paso el más importante de ellos, o sea el que corresponde a la esencia del valor, a su concepto y definición, a sus categorías constitutivas, a su demarcación problemática, su metodologfa dialéctica y configuración sistemática. Dicho problema es, en términos generales, el que corresponde al tratamiento universal de los valores y, en su propia generalidad, los planteamientos no pueden ser resueltos por las ciencias particulares que se ocupan de la especificación y realización material de los valores mismos.

El primer punto que sale a debate es el concerniente al concepto universal del valor, el cual figura entre los que discute la filosofía contemporánea. Es cierto que desde la antigüedad se habla de los valores, como es el caso de las doctrinas que se refieren a la verdad, la belleza, la justicia, la bondad, la santidad, etc., pero la referencia histórica atañe directamente a la opinión sostenida en cada caso y recae en la doxografía subjetivista inherente a toda la filosofía acrítica. No existe en ella una doctrina expresa de los valores, un concepto universal que denote no sólo la conciencia de realización en los hechos culturales, sino la autoconciencia critica axiológica que constituye un problęma privativo de la filosofía a partir del siglo xix, en cuyos mediados surgen las primeras corrientes axiológicas de la modernidad.

Si en toda la filosofía anterior se habían manejado con holgura los conceptos del ser, las ideas y la vida, la justificación del valor no sólo como un problema fundamental sino distinto de los demás, debió producirse en primer término por el señalamiento del valor con respecto a los otros conceptos axiomáticos y, además de ello, justificarse como el más amplio de los problemas, que es precisamente cual yo lo entiendo. Establecer ese distingo equivale en cierto modo a definir el valor por lo negativo, diciendo lo que no es, aunque 
también encontramos que el valor no equivale a los otros planteamientos aporéticos, pero los subsume bajo su propia extensión, de suerte que al afirmar el distingo del valor con respecto a los otros axiomas se hace también la inclusión correlativa, que deriva de la subsunción aporética y constituye la clave en la evolución histórica de la filosofía.

\section{La nueva metátesis}

En estas condiciones, lo más probable es que se esté operando una nueva metátesis en la filosofía moderna; el análisis que acabo de efectuar es sólo un índice de la gran transformación que impele hacia una tarea cuyo contenido axiológico podrá convertirla en ciencia particular o en un grupo de ciencias que representarían cuando menos el aspecto material de la verdadera axiología. Los ejemplos que he señalado demuestran, en los casos más conspicuos de los valores clásicos, cómo las diversas ciencias particulares disputan y en cierto modo arrebatan a la axiología filosófica su original atribución especulativa y la trasladan a la axiologia cientifica bajo la égida de la determinación concreta, mediante el poderoso auxilio de las ciencias particulares en cuyo traslado consiste precisamente la exégesis que considero indispensable para una correcta interpretación de la historia filosófica frente a la historia científica y cultural, ubicando la crisis del momento en las coordenadas expuestas por los anteriores ejemplos. De ellos se desprende, en síntesis, que la filosofía tien eahora pocos recursos para establecer en qué consisten los valores reales, mientras la ciencia los arbitra cada vez en mayor grado.

Así, la única verdad que cuenta realmente para la determinación positiva del ser, es la producida por los hombres de ciencia, que cuentan con el instrumental más abundante para llevar a cabo eficazmente la tarea. Los moralistas ideológicos no tienen buenas perspectivas de triunfo en su afán por establecer la forma pura de la conducta, desde el momento que ésta queda sujeta a la influencia de los factores operativos y es abordada desde su base por un gran número de disciplinas que relativizan, tanto al concepto filosófico de la moral como al valor de la conducta. Por último, los estéticos filosóficos que inventan "desde arriba" lo que debe ser la belleza pura, encontrarán graves reparos a sus teorías en el terreno artístico; si lo violentan no podrán permanecer como doctrinas estéticas del arte, y si no lo violentan es porque coinciden con él, en cuyo caso deberán prestar oído atento a lo que digan los especialistas en la materia, que para tal efecto son el historiador, el crítico y el técnico del arte.

\section{El pragmatismo axiológico}

Tratándose de otros valores, como puede ser, por ejemplo, el económico, 
no vemos cómo pudiera intervenir alguna pretendida filosofia económica que se opusiera a la ciencia económica en la tarea que acometen los especialistas para explicar el respectivo valor en el proceso del desarrollo social, sujeto a una serie de factores constitutivos que requieren la participación activa de numerosas disciplinas sociales para ser correctamente evaluados. El factor económico se traduce en el valor económico mediante la relación determinante de los procesos sociales, que es una relación causal-eficiente y no formal-ideológica, por cuyo motivo la economía se erige como la ciencia de la eficiencia y abarca todo el campo de las disciplinas sociales, al margen de cualquier ideología o filosofía especulativa. Otro tanto puede afirmarse de los valores históricos, o sean los prototipos que imperan en cada sociedad durante cierto tiempo; hablando en rigor, no existen los valores históricos propiamente dichos, sino la vigencia histórica de los valores, en la irreductible temporalidad o espaciotemporalidad que caracteriza a toda suerte real, de modo que la historicidad de los valores representa la confirmación básica y dinámica de su realidad.

La tarea que atañe a la valoración comparada de las épocas y las colectividades, inherentes al hito de la filosofía de la historia, se traduce en una problemática original donde se realizan incontables estudios tendientes a establecer los modelos axiosociohistóricos que permitan explicar en lo general y en lo particular el sentido de la vida, de las realidades e instituciones de cada época y lugar, pues la intervención de la ciencia no se limita a la verificación problemática o descriptiva - como frecuentemente se interpreta la faena histórica-, sino tiende a erigirse en calidad de una ciencia explicativa $o$ asertórica, y en último término, normativa o apodíctica, por la cual se manejan los problemas de los valores sociohistóricos de modo concreto y al mismo tiempo doctrinario.

Esta consideración se aplica también a los valores políticos, que representan ideológicamente la incorporación de las supremas finalidades regulativas del convivio, por lo cual aparentemente contendrían una dosis superior de ideología, como en efecto se ha propuesto en el seno de la llamada "filosofía política"; ella podría acercarse al nivel filosófico propiamente dicho, mas no por eso deberá aceptarse que la política se traduce en una especulación inocente de las condiciones sociológicas, antropológicas e históricas que estipulan la vigencia concreta de cualquier doctrina, en cuya virtud se erige, precisamente, como doctrina política. En este caso, igual que en los demás, hablo de doctrinas y no de utopías; me parece que este señalamiento encarna el sentir de la moderna teoría política, que tiende cada vez más a la reafirmación de los valores pragmáticos con la correspondiente disminución de los valores ideológicos. 


\section{La axiologia formal}

Por lo que he expuesto, creo estar en condiciones de concluir en la fundada posibilidad de que la nueva metátesis de la filosofia material pudiera dejarla sin el más caro y quizá el último de sus problemas actuales; el examen y la explicación concreta de los valores. Cuando menos a nivel de particularidad especifica; parece muy difícil que se llegue a evitar la transferencia de la temática axiológica mediante la determinación particular de los valores culturales que, entendidos como elementos de acción en la vida real, se efectúa principalmente en la aplicación de las ciencias que, desde su respectivo ángulo epistémico, acometen con gran eficiencia la ingente obra determinativa. Una vez más, las ciencias naturales, las ciencias sociales y las ciencias antropológicas - todas las ciencias particulares - disputan a la filosofía su campo de acción y también una vez más ostentan las mayores posibilidades de enajenarlo, si no es que lo han hecho ya.

La consecuencia que comporta esta metátesis para forjar un concepto autónomo de la filosofía y de la investigación filosófica, es mayúscula; podria decirse, total. Es un hecho cada vez más reconocido que nuestra ocupación se encuentra en una crisis de la cual podría sobrevenir su real o virtual desaparición; empero, yo me inclino a pensar que ahora, como ha sucedido en otras ocasiones, no desaparecerá, aunque debamos reconocer la necesidad de que en ella se efectúe una profunda transformación en concepto y método que la llevara a convertirse probablemente en una disciplina formal, como ha ocurrido en anteriores avatares. El que estoy comentando ahora revela a la axiología material transformándose en ciencias particulares, puesto que son ellas las indicadas para establecer las condiciones específicas de realización en los valores de toda especie, mientras la axiología formal se avoca al problema de señalar, examinar y explicar comparativamente, cuáles son las polaridades y dimensiones, niveles y jerarquias, que asume el valor en los diversos sectores de la cultura, en los diferentes estratos sociales, en las diversas comunidades y épocas históricas, cumpliendo el complejo proceso de integración particular y general que recoge por antonomasia la metodología dialéctica.

Ahora bien, teniendo en cuenta que la axiologia material se manifiesta como una disciplina o un conjunto de disciplinas particulares, yo considero que la axiologia formal tenderá a producir las generalizaciones inherentes al concepto clásico del filosofar, fundamentalmente cuando apunta al concepto universal del mundo y de la vida que, obtenido por tan plurivalente conducto, será un concepto esencialmente axiológico, una cosmopsicovisión fundada en lo que significan los valores para la vida individual y colectiva, así como en la interpretación de los problemas tradicionalmente adjudicados al ser y la idea, que ahora se subsumen en términos de valor: el ser como 
valor, la idea como valor, la vida como valor, y lo que deriva de todo ello, la historia como recipiente conjuntivo e integrativo de valores.

Tal suerte de investigación supone como base el establecimiento de la axiologia comparada, que se canalizará activamente como axiologia formal - metodología axiológica; en otras palabras, ello significa que la filosofía cumple una vez más el designio de la metátesis material ejerciendo la funciór, autocrítica que se traduce en la fundamentación metodológica de los valores, con lo cual se refrenda la acepción conferida por la escuela crítica, a cuyos postulados me adhiero, de la filosofia en calidad de metodologia. Éste es quizá el mayor motivo por el cual encuentro justificable la adhesión a una postura como es la criticista, de carácter estrictamente formal, que equivale a denegar todas las posturas en su rígida acepción material y particular. Al sumarme a esta corriente he tratado de aplicar sus axiomas primarios en el problema del valor, suponiendo que ahí se encuentra la tarea última, por ser la más fecunda, que erigirse pueda en la reflexión contemporánea.

Por todo ello señalo el alcance que en nuestra tarea puede tener, y tiene de hecho, la investigación filosoffica que, en cuanto investigación axiológica, salvará las confusiones surgidas en otras épocas respecto a la heterogénea y contradictoria modalidad del filosofar en su abstractiva asunción universal frente a las disciplinas particulares con las cuales ha disputado continuamente su objeto en el compulso de la reiterada aporía que conlleva una fase crítica en cada etapa evolutiva; la época contemporánea origina la nueva metátesis axiológica que me ha ocupado ahora, al poner de relieve en toda su gran importancia las consecuencias que reviste una profunda crisis que, a mi criterio, se ha definido claramente como hipotética conclusión y pretendido desenlace de la filosofía como axiología, entendida en su más correcta acepción: como axiología formal.

Miguel Bueno

Instituto de Investigaciones Filosóficas

Universidad Nagional Autónoma de México 